\title{
ORIGINAL
}

\section{EVALUACIÓN CUALITATIVA DE UNA CAMPAÑA DE PROMOCIÓN DEL USO DEL PRESERVATIVO EN LA POBLACIÓN ADOLESCENTE Y JUVENIL DE LA COMUNIDAD DE MADRID}

Luis Seoane Pascual

Servicio de Educación Sanitaria. Instituto de Salud Pública de la Comunidad de Madrid. Consejería de Sanidad.

\section{RESUMEN}

Fundamentos: El Programa de Normalización del Uso del Preservativo, viene desarrollando desde noviembre de 1998 distintas actividades de promoción del uso del preservativo entre la población adolescente y juvenil. Todas ellas pivotaban sobre una acción específica destinada a favorecer el acceso: la venta en establecimientos de farmacia de preservativos a bajo precio destinados a la población adolescente y juvenil. El objetivo de esta investigación fue la evaluación de los resultados desde el punto de vista de su eficacia persuasiva para modificar comportamientos de riesgo.

Métodos: La metodología cualitativa empleada fue la habitual en el contexto de los estudios de mercado y opinión: el post-test cualitativo sobre la base de entrevistas abiertas con farmacéuticos y jóvenes. Se efectuaron 7 entrevistas con farmacéuticos y 14 con jóvenes de ambos sexos, de edades comprendidas entre 17 y 19 años, con un comportamiento sexual activo y residentes en zonas de riesgo desde el punto de vista de la incidencia del sida.

Resultados: Se identificaron dos factores de riesgo fundamentales: a) El desarrollo de estrategias por parte de determinados varones con éxito sexual, siempre en el contexto de relaciones sexuales inestables, de evasión del uso del preservativo con el objeto de obtener un placer más pleno, ante una evaluación previa del grado de riesgo de la relación; la pastilla post-coital, como método anticonceptivo alternativo, aparece implicada en tales estrategias con bastante asiduidad. b) La inhibición de la compra del preservativo en la farmacia, que impide el desarrollo de una conducta previsora y que, por tanto, genera directamente la aparición de situaciones de riesgo.

Conclusiones: La evaluación de la campaña ha sido positiva desde el punto de vista puramente informativo. No se han observado, sin embargo, elementos que permitan actuar eficazmente en contra del principal factor de riesgo detectado, el desarrollo de estrategias de evasión del uso del preservativo con el objeto de obtener un placer más pleno y la sanción por parte del grupo de esos comportamientos. De cara al segundo factor de riesgo, el de la inhibición de compra, no se ha advertido eficacia sensible en la propuesta de venta de preservativos a bajo precio en farmacias.

Palabras clave: Adolescencia. Investigación cualitativa. Enfermedades de transmisión sexual. Evaluación. Programas de salud. Preservativos.

\section{Correspondencia:}

Luis Seoane Pascual

Instituto de Salud Pública de la Comunidad de Madrid.

Aduana 29. 28005 Madrid

Correo electrónico: luis.seoane@madrid.org

\section{ABSTRACT}

\section{Qualitative Assessment of a Campaign} Promoting Condom Use among a Teenage and Young Adult Population in the Community of Madrid, Spain

Background: The Program for Standardizing Condom Use has been carrying out different activities to promote condom use among the teenage and young adult population since November 1998. All of these measures have revolved around a specific measure aimed at making condoms more readily available, that is, the sale of condoms to the teenage and young adult population in pharmacies at low prices. The objective of this research was to assess the results from the standpoint of the persuasive effectiveness of these measures as related to modifying unsafe practices.

Methods: The qualitative methodology employed was the customary methodology within the context of market and opinion studies, that is qualitative after-test on the bases of open interviews with pharmacists and youths. A total of 7 interviews were held with pharmacists in addition to 14 interviews with sexually active young people of both sexes within the 17-19 age range residing in unsafe areas from the standpoint of AIDS incidence.

Results: Two main risk factors were identified: a) The approaches taken on the part of certain sexually successful males, always within the context of casual sexual relations, of avoiding condom use for the purpose of greater sexual pleasure in view of a prior assessment of the degree of risk involved in the relations in question; the post-coital pill as an alternative means of contraception seems to be involved very often in such approaches. b) The inhibition of purchasing condoms in the pharmacy, which prevents taking due precautions and, therefore, directly gives rise to unsafe situations arising.

Conclusions: The campaign in question has been assessed as being positive from the purely informative standpoint. No aspects have however been encountered which afford the possibility of effectively countering the main risk factor detected, the approaches taken of avoiding the use of a condom for the purpose of achieving greater sexual pleasure and the sanctioning of these practices on the part of the group. As regards this second risk factor, that of inhibition regarding purchase, no appreciable effectiveness has been noted as regards the sale of condoms at a low price in pharmacies.

Keywords: Teenage years. Qualitative research. Sexually-transmitted diseases. Assessment. Health programs. Condoms. 


\section{INTRODUCCIÓN}

El Programa de Normalización del Uso del Preservativo ${ }^{1}$, en el que colaboran aproximadamente una treintena de instituciones relacionadas con la salud y la educación en la Comunidad de Madrid, viene desarrollando desde noviembre de 1998 distintas acciones de promoción del uso del preservativo entre la población adolescente y juvenil. Teniendo como objetivo fundamental la protección de chicos y chicas de las enfermedades de transmisión sexual (E.T.S.) y de embarazos no deseados, dicho Programa se centra fundamentalmente en la prescripción del uso de preservativo en las relaciones sexuales juveniles como método anticonceptivo profiláctico de primera elección. A tal fin se desarrollaron, entre otras, una serie de acciones focalizadas sobre los establecimientos de farmacia, en tanto que lugares de venta privilegiados del preservativo. Cabría destacar las siguientes acciones: a) concienciación del farmacéutico y/o del empleado de farmacia en su función de prescriptor del uso del preservativo a la población juvenil y b) demanda de colaboración y de adhesión de esos mismos profesionales a las acciones concretas del Programa de Normalización que les incumben directamente; fundamentalmente: venta alternativa en establecimientos de farmacia de cajas de preservativos de tres unidades a bajo precio dirigidas específicamente a la población juvenil, y promoción directa o indirecta de dichos preservativos a los jóvenes desde los establecimientos, cuya marca externa era la del lema general de la campaña Si te lías... úsalo.

La demanda de la investigación, cuyos resultados se resumen en este artículo, y que fue encargada a una empresa de investigación de mercados y opinión, se centró fundamentalmente en la evaluación de las acciones de comunicación del Programa de Normalización realizadas específicamente sobre los establecimientos de farmacia, considerando las dos partes implicadas en ellas, los jóvenes, en tanto que «objetivo» privilegiado de las acciones comunicativas y como «consumidores» directos de los preservativos propuestos, y los farmacéuticos y los empleados de farmacia en tanto que «prescriptores»y en tanto que vendedores directos de un tipo de preservativos. Ello suponía el considerar la relación que se establece entre el joven y el profesional de farmacia en la venta del preservativo, y su evaluación con relación a la pauta ideal desde el punto de vista de la consecución de los objetivos de salud pública presentes en el Programa de Normalización del Uso del Preservativo $^{1}$.

La evaluación de una campaña comunicativa, como la que opera como pretexto del estudio, suele realizarse en el ámbito privado desde dos tipos de investigaciones estandarizadas: el post-test cuantitativo, cuyo objeto es obtener una medida del cambio de comportamiento efectivo atribuible a una campaña; y el post-test cualitativo, cuyo objeto es determinar el grado de persuasión de una campaña de cara a la modificación de los comportamientos que se pretendían corregir.

Un post-test cuantitativo ofrece resultados «objetivos» que pueden ser útiles para evaluar la eficacia real de la campaña. Sin embargo, queda como resto la respuesta a preguntas vitales para orientar la estrategia comunicativa global: ¿Por qué la campaña ha funcionado o no ha funcionado? ¿Qué elementos han sido decisivos en una u otra dirección? La posibilidad de establecer correcciones tácticas o estratégicas en una campaña de comunicación de cara a optimizar sus resultados es lo que justifica prácticamente el recurso a técnicas cualitativas de investigación. Operativamente, un post-test de campaña cualitativo debe seguir los siguientes pasos:

1. Aislar los móviles principales de la conducta que se pretende corregir. En nuestro caso se trataba de identificar las razones justificativas de la conducta de riesgo de efectuar relaciones sexuales sin preservati- 
vo. Y no existe otro camino para llegar a esa identificación que preguntar, como se hizo, a los propios adolescentes implicados en esas conductas de riesgo. La recurrencia de móviles similares en distintos sujetos nos da una garantía de que estamos ante una pauta, o pautas motivacionales esenciales asociadas a la conducta de riesgo.

2. El segundo paso lógico consiste en formalizar comprensivamente esa conducta a partir de los móviles mentados. Fundamentalmente se trata de reconstruir la conducta típica desde un esquema finalista, de adecuación racional entre medios y fines ${ }^{2}$. Esta reconstrucción nos permite, por lo menos intuitivamente, identificar los posibles puntos débiles del discurso del sujeto, de la justificación de su conducta, o los puntos débiles de los intentos externos de influir en su conducta.

3. Un tercer paso consiste en enfrentar al sujeto a la discusión de elementos de la campaña en cuestión y de otras campañas similares. Se trata de una reconstrucción en acto de la capacidad de persuasión de elementos de comunicación que pretenden influir en la conducta del sujeto. A partir de los pasos anteriores estaríamos en disposición de explicar las reacciones que se dan de hecho ante la intervención comunicativa. Esta capacidad de explicación del método es lo que le faculta para proponer correcciones ulteriores sobre la campaña de comunicación en cuestión.

\section{SUJETOS Y MÉTODO}

Se realizó una investigación cualitativa de carácter exploratorio y limitada en sus dimensiones. Se efectuaron:

- 7 entrevistas abiertas con profesionales de farmacia, de ambos sexos, implicados y no implicados, al 50\%, en el programa de normalización;

- 14 entrevistas abiertas con jóvenes de edades comprendidas entre 17 y 19 años, de ambos sexos, de clase social media baja y baja, que tuvieran relaciones sexuales con relativa asiduidad, residentes en zonas de riesgo desde el punto de vista de la incidencia del sida por transmisión sexual.

La demanda de una investigación de mercados cualitativa se mueve siempre dentro de los parámetros prácticos de una intervención comunicativa sobre sujetos sociales, sobre grupos sociales. La intervención comunicativa en este caso se efectuaba, como se dijo, sobre los adolescentes y jóvenes de la Comunidad de Madrid con la intención manifiesta de modificar comportamientos de riesgo, entendiendo por tales el no uso del preservativo en las relaciones sexuales.

\section{RESULTADOS}

El estudio se ha focalizado estratégicamente en la exploración de los factores de riesgo en el tipo de sexualidad propio de la adolescencia: una sexualidad de iniciación, estructuralmente promiscua —en la medida en que no suele cursar con relaciones de pareja demasiado estables-, débilmente premeditada, condicionada en muchos casos desde reglas marcadas desde el propio grupo adolescente y bastante poco permeable al control y la supervisión adultas. Se trata evidentemente de una etapa pasajera; muchos de las actitudes comportamientos reflejados en el estudio desaparecerán rápidamente conforme el sujeto vaya evolucionando en su proceso de maduración.

Se observan en las entrevistas dos tipologías comportamentales claramente diferenciadas al respecto del uso del preservativo: los que siempre lo utilizan y no se plantean, por lo menos a priori, ninguna excepción al respecto, y los que lo utilizan sólo a veces, ante la evaluación del grado de riesgo de una determinada relación.

No se pueden ofrecer, como es obvio, datos cuantitativos acerca de la magnitud de ambos colectivos, pero sí que es fácil identi- 
ficar una relación clara entre el grado de asunción de la norma del uso del preservativo y el grado de asunción de otras normas adultas. En este sentido, conforme el sujeto se acerque más al tipo extremo de joven sin metas personales definidas, fracasado en sus estudios, con débil control paterno y volcado en el hedonismo de la juerga es más normal que esté en el segundo grupo que en el primero.

El no uso puntual del preservativo, cuando se detecta, está menos relacionado con un problema de información que con un problema de perversión. El principal enemigo actualmente de la extensión del uso del preservativo es la expectativa de goce que se ha instalado tras la ausencia de su utilización. El no uso del preservativo en el adolescente es menos un error, o un desconocimiento de las posibles consecuencias, que un acto conscientemente orientado a la búsqueda de un goce en un ámbito de riesgo calculado.

$\mathrm{Y}$ en este contexto de acto conscientemente orientado, se observan indicadores claros de que la pastilla post-coital (asociada o no a otras prevenciones tradicionales) se está convirtiendo en un recurso anticonceptivo habitual para poder tener relaciones sexuales plenas sin preservativo y, por tanto, para poder acceder con un mínimo riesgo al goce narcisista previsto.

Esta conducta perversa se manifiesta como típicamente masculina. El goce del que se habla es siempre el goce del varón; las chicas en ningún momento plantean objeciones relacionadas con el placer que ellas mismas sienten; si acceden a tener relaciones sin preservativo siempre resulta ser una concesión al goce masculino.

Por el contrario, lo que se advierte con facilidad en este tipo de chico es el desarrollo de estrategias (de seducción) para conseguir que la chica disuelva sus resistencias y acepte tener relaciones sexuales sin preser- vativo. A la vista de los discursos, parecen ser precisamente los chicos con mayor éxito sexual los que suelen abundar más en este tipo de estrategias o, por lo menos, los que aparentan tener más facilidad para llevarlas a cabo satisfactoriamente.

De lo dicho se deduce que en la lucha contra el comportamiento perverso de no utilizar preservativo es vital contar con la colaboración de las chicas. En la medida en que éstas se juegan más (asumen mayor riesgo) en la no utilización del preservativo y en la medida en que tampoco advierten de igual manera que el chico una diferencia placentera en hacerlo o no sin él, es razonable proponerles un papel más activo. Es norma en el caso de las chicas considerar que el preservativo es responsabilidad exclusiva del chico y el mostrar un pudor excesivo a la posibilidad de llevarlo ellas mismas en su bolso.

En cierto sentido puede decirse que el grupo adolescente actúa convalidando este tipo de comportamientos perversos. Como sucede con otros comportamientos «arriesgados», quien los efectúa puede incrementar su valor y su protagonismo dentro del grupo.

El acceso del adolescente a la compra del preservativo sigue siendo también un problema. Aunque previsiblemente el mayor problema de fondo esté en el apuntado de la perversión ante su uso, es obvio que la dificultad de acceso puede convertirse en coartada para no llevar preservativo y, por tanto, para tener relaciones sin él.

Y es obviamente un problema el que sea la farmacia el único establecimiento donde el adolescente puede acceder a un preservativo con garantías. La posibilidad de superar el «corte» de pedir el preservativo al profesional de farmacia es prácticamente imposible a ciertas edades, y es más difícil todavía en el caso de la chica que en el del chico. Con este problema todo queda hipotecado muchas veces a la posibilidad de que 
alguien en el grupo lo lleve en el momento oportuno, imposibilitándose la instalación de un comportamiento previsor.

Hay que entender que la superación del corte a ir a la farmacia implica madurez y asunción por parte del joven de su propia sexualidad. El joven que va naturalmente a la farmacia debe tener previamente una actitud especial que sólo se evidenció en los casos en que se pudo constatar un proceso formativo previo con la participación de padres y/o educadores, que la mayoría de las veces no se daba. La falta de esa formación necesaria, que excede los esfuerzos simples de información, es con claridad una asignatura pendiente.

Es muy difícil que el chico o la chica accedan a la farmacia e instalen un comportamiento previsor en la compra del preservativo si previamente deben de vivir su sexualidad como algo prohibido y culpable. Y lo normal, a la vista de lo que nos dicen en las entrevistas, es que el adolescente viva su iniciación sexual completamente en secreto, a escondidas de sus padres y sopesando el riesgo de trauma familiar que puede significar su descubrimiento. No debemos creer, en referencia a la sexualidad adolescente, que la situación actual sea de tolerancia. Por lo menos, si consideramos el discurso de los jóvenes acerca de la actitud de los progenitores, la norma por parte de los padres es la de no querer afrontar ni dar credibilidad a la posibilidad de una sexualidad precoz en los propios hijos.

\section{DISCUSIÓN}

La visión, necesariamente restringida, de la sexualidad adolescente en la Comunidad de Madrid que nos ofrecía el estudio permitía sacar conclusiones medianamente positivas en lo que respecta a la utilización del preservativo, congruentes con las de otros estudios empíricos similares ${ }^{3}$ : el preservativo está prácticamente siempre mediando como deber en la sexualidad adoles- cente. Sin embargo, en la medida en que la norma es conocida y reconocida como deber, se identifican estrategias típicamente adolescentes de perversión de esa norma, que claramente desembocan en comportamientos de riesgo en lo que respecta a la propagación de enfermedades de transmisión sexual.

Del análisis concreto sobre los elementos del Programa de Normalización del Uso del Preservativo $^{1}$ en la Población Joven de la Comunidad de Madrid que han podido ser testados, no se derivan conclusiones demasiado positivas, si la evaluación se realiza desde posibles planteamientos estratégicos más amplios y más avanzados que los de una simple campaña informativa.

Como pura campaña informativa la evaluación es positiva, tanto en la forma como en el fondo. Se dice lo que se tiene que decir, y se dice además de una forma atractiva y atrayente para el joven, incorporándose, además, innovaciones interesantes, como es el teléfono gratuito de información sexual.

El problema detectado, en cualquier caso, no es intrínseco a la campaña, sino extrínseco. Lo que se ha observado es la indiferencia general ante los mensajes de estas campañas informativas, sobre todo en aquellos jóvenes que no están previamente convencidos y que, por tanto, corresponden al tipo de los que utilizan el preservativo sólo a veces. La capacidad de esta campaña y de otras semejantes para influir en el comportamiento de los adolescentes en este tema es muy limitada, fundamentalmente porque lo que se dice ya se conoce: se sabe que hay que utilizar preservativo y se sabe por qué.

Si se acepta la hipótesis inicial de que el no uso del preservativo se debe más a una conducta perversa - asimilable, por tanto, a otras conductas perversas típicamente adolescentes, como el consumo de alcohol o de drogas ${ }^{4}$ - que al desconocimiento, se puede entender por qué esta campaña resulta parcialmente ineficaz. La perversión, a diferen- 
cia de la subversión, es un comportamiento parcial reactivo que nunca supone el cuestionamiento global de la norma. Se trata de un coqueteo con lo prohibido, en el cual se asume el menor riesgo posible, pero que produce una sensación agradable de omnipotencia y que, en el caso típico del adolescente, supone una vía privilegiada de autoafirmación frente a la dependencia de la sociedad adulta ${ }^{5}$. Lo propio de la conducta perversa es actuar contra la norma; y ante esa evidencia, la repetición de la norma lo único que consigue es reforzar el comportamiento desviado. No porque se grite más se consigue que el adolescente haga más caso; es más, previsiblemente hará menos caso conforme reciba más veces el mensaje.

El comportamiento perverso tiene su racionalidad, por lo que una táctica posible consiste en intentar desmontarla. Y hay que aceptar que es racional que el adolescente recurra a la pastilla post-coital: es su forma de hacer lo contrario de lo que le dicen pero sin correr excesivos riesgos ${ }^{3}$. Si le problematizamos esa salida informándole más de sus riesgos es posible que reconsidere su actitud, simplemente por prevención.

Otra vía posible de desmontar el comportamiento perverso podría ser la de extender la información a todo el ámbito de enfermedades venéreas, comunicando la probabilidad de contraerlas y el riesgo final que se corre. Prácticamente sólo el sida aparece como argumento higiénico para utilizar preservativo, pero el sida es la enfermedad más improbable, y con ello cuenta el joven a la hora de establecer su estrategia subversiva ${ }^{6}$.

Hay que admitir que el sida día a día está perdiendo fuerza para condicionar el comportamiento sexual adolescente. Si ya previamente se observaba en el adolescente una gran dificultad para considerar a los colegas del grupo como potenciales transmisores de la enfermedad ${ }^{7}$, la actual reducción de la visibilidad pública del sida, relacionada en gran medida con los éxitos de la estrategia global de prevención, necesariamente debe de actuar reduciendo sensiblemente la impresión de riesgo. Quizá se esté en un momento en que para proteger a los adolescentes de esta enfermedad haya que trascender el planteamiento habitual de informarle sin más de sus riesgos.

Todo lo que permita favorecer la interiorización de la norma por parte del joven debe de resultar positivo. Para ello es indispensable que éste aparezca como el protagonista de la comunicación, y no como simple destinatario pasivo e ignorante frente a un mensaje que le transmiten instancias adultas. Creemos que pueden ser más eficaces campañas de comunicación con protagonistas juveniles y planteadas en contextos juveniles que campañas como la que nos ocupa, en las cuales el receptor no tiene capacidad para identificarse ni con personajes ni con situaciones.

El esfuerzo debería de estar concentrado en desautorizar comportamientos frecuentes de riesgo y las actitudes que los provocan, y refrendar las contrarias. Vía identificación con personajes y situaciones concretos, se le deben ofrecer al adolescente armas para contrarrestar el comportamiento perverso del grupo y para salir indemne de las situaciones de riesgo. El objetivo principal sería conseguir que el adolescente tome las riendas de su propia sexualidad a pesar de, y en gran medida, en contra de los mensajes de prohibición que recibe del mundo adulto. Sólo en un contexto semejante se puede conseguir una auténtica normalización del uso del preservativo. Sólo se puede normalizar el uso del preservativo si la propia sexualidad adolescente deviene normalizada. A fin de cuentas, una intervención formativa exige dejar de una vez por sentado que el adolescente conoce el riesgo, y empezar a trabajar en el terreno más complicado, el de las dificultades que tiene aquél apara asumir el comportamiento racional consecuente.

Considerando particularmente a las chicas, una intervención «feminista» sobre el colectivo de las adolescentes con relación al 
hecho de llevar preservativo podría ser una acción eficaz en el contexto de las campañas generales de concienciación e información. A todas luces, considerando el tipo de sexualidad esporádica y furtiva del adolescente, no es suficiente con que ellas digan «no» cuando no hay preservativo. Hay que conseguir que también lo lleven, porque ese «no» puede ser muy fácilmente trocado en "sí»" cuando lo que está en juego es la seducción de un chico «tentador».

La evaluación de esa otra parte del Programa que consiste en la promoción para adolescentes de cajas de preservativos a bajo precio de venta en farmacias ha sido bastante negativa. En primer lugar porque el precio no es una razón más que muy secundaria de inhibición a la compra del preservativo, por lo menos considerando el canal farmacias. Si el joven no acude a la farmacia a comprar preservativos no es porque éstos le resulten demasiado caros, sino porque no puede superar el corte que le produce la situación. En el contexto de la farmacia, no es posible generar la demanda de aquellos que no se acercaban a la farmacia simplemente con el gancho de un precio más reducido. Pero una vez que el joven supera su inhibición a la compra en la farmacia, tampoco muestra un interés especial por un preservativo de marca desconocida. Prefiere, aunque pague más, un preservativo de las grandes marcas, que le ofrece las máximas garantías. Ante esta situación poco podía hacer el farmacéutico, por mucha buena voluntad que pusiese.

Por otra parte, hay que ofrecer al adolescente modos de acceso alternativos a la farmacia que le permitan superar su «corte».

La alternativa de las máquinas en discotecas, pubs y gasolineras no permite que se desarrolle un comportamiento previsor, debido al déficit de garantía que cualquier adolescente le asigna. Sirven sólo como solución de urgencia.

Las máquinas de farmacia, sin lugar a dudas, han sido la solución reciente más efi- caz. Sería positivo que se divulgara esa alternativa y también el proponer otras alternativas de similar o mayor eficacia, como la venta de preservativos en colegios.

Sugerir el posible acceso a otras alternativas como supermercados o perfumerías, y sancionar su garantía, sería también otra medida razonable.

El problema que se pretendía resolver era imposible de abordar desde el canal farmacias. Para hacer más accesible el preservativo al joven hay que proponerle otros lugares de compra. La iniciativa reciente de proponer la venta de estos preservativos en máquinas expendedoras en lugares públicos creemos que es más ajustada a la naturaleza del problema.

Queda por señalar que el estudio tenía una finalidad fundamentalmente práctica: evaluar, al modo de una investigación de mercados, una campaña comunicativa destinada a promocionar el uso del preservativo en la población adolescente y juvenil de la Comunidad de Madrid. En modo alguno, por tanto, se trataba de una investigación global, con pretensiones de representatividad, de la sexualidad de los adolescentes y jóvenes de la Comunidad de Madrid. Cualquier intento de extender los resultados fuera del contexto de su producción supone una operación ilegítima.

\section{BIBLIOGRAFÍA}

1. Programas de Salud Pública. Memoria 1999. Madrid: Consejería de Sanidad; 2000. Documentos técnicas de Salud Pública núm. 63.

Programas de Salud Pública 2000. Madrid, Consejería de Sanidad; 2000. Documentos técnicos de Salud Pública núm. 62.

2. Weber M. Conceptos sociológicos fundamentales. En: Economía y Sociedad. 3. ${ }^{\mathrm{a}}$ ed. Madrid: Fondo de Cultura Económica; 1993. p. 9-45.

3. Conde F. El mundo de las relaciones personales de los jóvenes madrileños. Investigación cualitativa previa del programa de normalización del pre- 
servativo. (edición mimeografiada). Madrid: Consejería de Salud; 1999. p. 122.

4. Peinado A, Pereña F y Portero P. La cultura del alcohol entre los jóvenes de la Comunidad de Madrid. Madrid: Consejería de Salud; 1993. Documentos Técnicos de Salud Pública núm. 9.

5. Ibáñez J. Más allá de la Sociología. El grupo de discusión, técnica y crítica. $1 .^{\mathrm{a}}$ ed. Madrid: Siglo XXI; 1979. p.205.
6. Soto Más F, Lacoste Martín J, Papenfuss L, Gutierrez León A. El modelo de creencias de salud, un enfoque teórico para la prevención del Sida. Rev Esp de Salud Pública 1997; 71: 335-341.

7. Conde F. Las Representaciones Sociales sobre la Salud de los Jóvenes Madrileños. Madrid: Consejería de Sanidad y Servicios Sociales; 1997. Documentos Técnicos de Salud Pública núm. 45. p. 67-68. 